
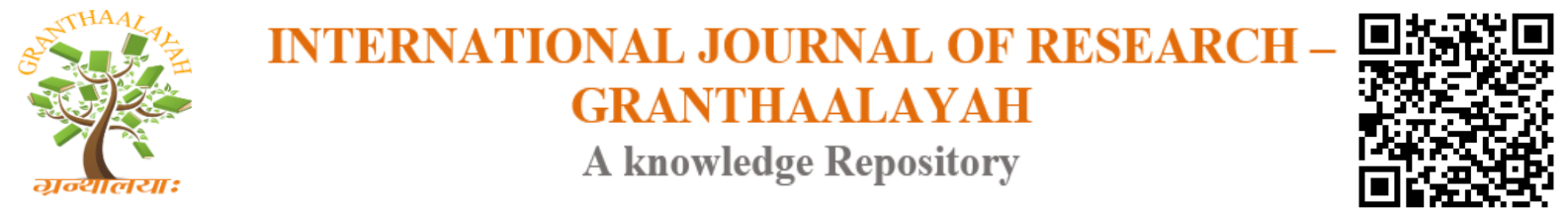

Science

\title{
WATER AND WASTEWATER OPTIMIZATION IN MULTIPLE CONTAMINANTS NETWORK USING WATER PINCH TECHNOLOGY
}

\author{
R.Sasikala ${ }^{* 1}$ \\ ${ }^{* 1}$ M.Sc., M.Phil., B.Ed., Head \& Associate Professor, Department of Computer Science, \\ National College(Autonomous), Trichirapalli, Tamilnadu, India
}

\begin{abstract}
Data mining is the process of analyzing data from different perspectives and summarizing it into useful information. Data mining is applied to find patterns to help in the important tasks of medical diagnosis and treatment. In this paper, we present a sensor to measure water level in rivers, lakes, lagoons and streams. For such purpose and to prove our concept, we designed a pilot project through a micro-model that is constructed with a water level measurement sensor based on a simple open circuit that closes when in contact with water and experimentally tested into a water container under a controlled environment. The indicator for acidity, alkalinity, or basic is known as the PH value. A PH value of 7 means a substance is neutral. The lower value indicates acidity, and a higher value is a sign of alkalinity. Side effects that may occur after drugs are added to Bacteriostatic. Water includes fever, abscess formation, venous thrombosis or phlebitis, tissue death, and infections. We are going to implement weka tool to compare the time execution, frequency and an execution process by using a genetic algorithm. Here we used the Ultrasonic Sensor technique to measure the water level. Ultrasonic sensors are based on the measurement of the properties of acoustic waves with frequencies above the human audible range, often at roughly $40 \mathrm{kHz}$. They typically operate by generating a high-frequency pulse of sound, and then receiving and evaluating the properties of the echo pulse. In this study, the principle of water pinch technology, the optimization of water, steam allocation network have been studied and minimization of freshwater utility and wastewater generation in one of the petroleum refineries of Iran is taken into consideration. In order to research in this field and simplify the relevant calculations, an algorithm was developed and applied for reforming the network. Finally, the micro-model is tested by experimental tests under a controlled environment and satisfactory results are obtained. The experimental evaluation results showed a $99.2 \%$ of accuracy and which proves this mechanism effective and reliable in water optimization.
\end{abstract}

Keywords: PH Value Measurement; Bacteriostatic Water; Weka Tool; Ultrasonic Sensor; Genetic Algorithm.

Cite This Article: R.Sasikala. (2017). "WATER AND WASTEWATER OPTIMIZATION IN MULTIPLE CONTAMINANTS NETWORK USING WATER PINCH TECHNOLOGY." International Journal of Research - Granthaalayah, 5(8:SE), 1-7. https:// doi.org/10.29121/granthaalayah.v5.i8(SE).2017.2237. 


\section{Introduction}

Data Mining is a knowledge mining process. It is an interdisciplinary subfield of computer science. It is the computational process of discovering patterns in large data sets involving methods at the intersection of artificial intelligence, machine learning, statistics and database systems.

Global water consumption. Water is a valuable natural resource, which is used for different purposes in daily life and various sectors, namely, domestic, agricultural, and industrial. Average global water consumption within those sectors varies, and it depends of the development level of countries.

In order to address the synthesis problems of separate and total water networks, different tools and methods have been proposed. Here Water Level is identified by PH Level Measurement of water were been based on acidity, alkalinity, or basic. PH level measurement 7 becomes normal otherwise it is to be resolved. To show the best result genetic algorithm was implemented to find out the processing time, Frequency level measurement using Ultrasonic Sensor technique.

The main research challenges have been devoted to systematically exploring all water integration opportunities within the process in order to achieve solutions with a reduced water usage and wastewater generation. It is important to mention that the synthesis problem of an overall or total water network is more complex, and this problem has been addressed fewer papers compared to the synthesis problem of separate networks (water using network or wastewater network)

\section{Literature Review}

\subsection{Global Optimization for the Synthesis of Integrated Water Systems in Chemical Processes}

In this paper, we address the problem of optimal synthesis of an integrated water system, where water using processes and water treatment operations are combined into a single network such that the total cost of obtaining freshwater for use in the water using operations, and treating wastewater is minimized. A superstructure, which incorporates all feasible design alternatives for water treatment, reuse and recycle, is proposed. We formulate this structure as a non-convex Non-Linear Programming (NLP) problem, which is solved to global optimality. The problem takes the form of a non-convex Generalized Disjunctive Program (GDP) if there is a flexibility of choosing different treatment technologies for the removal of the various contaminants in the wastewater streams. A new deterministic spatial branch and contract algorithm is proposed for optimizing such systems, in which piecewise under- and over-estimators are used to approximate the non-convex terms in the original model to obtain a convex relaxation whose solution gives a lower bound on the global optimum. These lower bounds are made to converge to the solution within a branch and bound procedure. Several examples are presented to illustrate the optimization of these integrated networks using the proposed algorithm. 


\subsection{Water and Energy Integration: A Comprehensive Literature Review of Non- Isothermal Water Network Synthesis}

Syntheses of non-isothermal water networks consisting of water-usages, wastewater treatment, and heat exchanger networks has been recognised as an active research field in Process Systems Engineering. However, only brief overviews of this important field have so far been provided within the literature. This work presents a systematic and comprehensive review of papers published over the last two decades and highlights possible future directions within this field. This review can be useful for researchers and engineers interested in water and energy integration within process water networks using systematic methods based on pinch analysis, mathematical programming, and their combination. We believe that this research field will continue to be active in the near future due to the importance of simultaneous optimising processes, water and energy integration for achieving profitability and sustainability within process industries.

\subsection{Water Network Optimization with Wastewater Regeneration Models}

The conventional water network synthesis approach greatly simplifies wastewater treatment units by using fixed recoveries, creating a gap for their applicability to industrial processes. This work describes a unifying approach combining various technologies capable of removing all the major types of contaminants through the use of more realistic models. The following improvements are made over the typical superstructure-based water network models. First, unit-specific short-cut models are developed in place of the fixed contaminant removal model to describe contaminant mass transfer in wastewater treatment units. Short-cut wastewater treatment cost functions are also incorporated into the model. In addition, uncertainty in mass load of contaminant is considered to account for the range of operating conditions. Furthermore, the superstructure is modified to accommodate realistic potential structures. We present a modified Lagrangean-based decomposition algorithm in order to solve the resulting nonconvex Mixed-integer Nonlinear Programming (MINLP) problem efficiently. Several examples are presented to illustrate the effectiveness and limitations of the algorithm for obtaining the global optimal solutions.

\subsection{Review of Optimization Models for Integrated Process Water Networks and their Application to Biofuel Processes}

This paper provides an overview of recent development in the area of optimal synthesis of process water networks in which a major goal is to reduce the freshwater consumption by the reuse and recycle of process and treatment streams. The recent models can globally optimize these networks through mixed-integer nonlinear programming techniques. We discuss the application and impact of these techniques to biofuel plants, which are known to consume large amounts of water

\subsection{Integrated Water Management Design Criteria Report}

The three-fold purpose of this project was to develop a product design criteria methodology to assess water saving products and systems; identify water products and systems that perform well against the design criteria; and comment on any potential commercial opportunities. Different 
products scored differently against different criteria, with the best overall 'score' coming from a combined 'system', comprising a low flow shower head, a water efficient washing machine, a 9,000 litre rain water tank and greywater reuse. Two potential commercial opportunities were identified; the installation of a relatively small 200 litre rainwater tank attached to the side of the house to supply toilet water only; and a 'modular' tank system where small storage blocks of 200 to 300 litres each could be connected up in irregular shapes to fit under decks, etc.

\section{Methodology}

\subsection{PH Value}

PH Value PH of 7 is neutral. A PH less than 7 is acidic. A pH greater than 7 is basic. The $\mathbf{p H}$ scale is logarithmic and as a result, each whole $\mathbf{p H}$ value below 7 is ten times more acidic than the next higher value. It all has to do with hydrogen ions (abbreviated with the chemical symbol $\mathrm{H}^{+}$). For simplicity, hydronium ions are referred to as hydrogen ions $\mathrm{H}^{+}$. In pure water, there are an equal number of hydrogen ions and hydroxide ions. The solution is neither acidic or basic. An acid is a substance that donates hydrogen ions.

\subsection{Data Set}

A collection of related sets of information that is composed of separate elements but can be manipulated as a unit by a computer. A data set is organized into some type of data structure. In a database, for example, a data set might contain a collection of business data like Calculating PH Value, Sensor Measurement, Frequency Calculation, Purification . The database itself can be considered a data set, as can bodies of data within it related to a particular type of information, such as sales data for a particular corporate department.

\subsection{Genetic Algorithms}

Genetic algorithm facts are as follows:

- Heuristic Search Algorithms Method based on evolutionary ideas of natural selection and genetics

- Provides efficient, effective techniques for optimization

- Useful when search space very large or too complex for analytic treatment

\section{Algorithm Key Concepts are as follows:}

1) Individual - Any possible solution

2) Genes-Attributes of an individual

3) Population - Group of all individuals

4) Search Space - All possible solutions to the problem

5) Chromosome - (set of genes) Blueprint for an individual

6) Fitness function- A function that assigns a fitness value to an individual

7) Genetic Operators:

- Reproduction (Selection)

- Crossover (or Recombination)

- Mutation-(Changing or Modifying) 
The steps involved in determination of optimal set of attributes are:

\section{Inputs:}

1) Mushroom database

2) Standard range of attributes (e.g. cholesterol, BP, etc.) from expertise

3) Rules generated from FP Growth \&GA.

\section{Initial Requirement:}

Dataset needs to be pre-processed for better decision making.

\section{Output:}

Optimal values of attributes i.e. the best item set algorithms Aprior or GA

\section{Size of Population:}

Suppose no. of attributes is N. Therefore initial population will consist of $\mathrm{N}$ chromosomes. But total size of population will be $2 \mathrm{~N}$ where next $\mathrm{N}$ will consist of newly generated population.

\section{Chromosome Initiation:}

Chromosomes will consist of one distinct value for every attribute. Hence size of chromosomes will be N. (Here attribute is gene)

\section{Fitness Function:}

In the proposed system, the fitness function calculates the optimal values of attributes based on three parameters:

- Actual value i.e. the gene value

- Deviation from normal values (the standard values obtained from expertise)

- Frequency of the value of an attribute

\section{Steps of Fitness Calculation:}

1) Check if gene value lies in the range given by Expert.

If no, then calculate the deviation from normal value using: Deviation = gene[i] value - Normal value, $0<=\mathrm{i}<\mathrm{N}$ Else Deviation $=0$

2) Calculate fitness of a gene using formula:

$=($ Frequency of occurrence of gene $[\mathrm{i}] *$ Deviation $) / \mathrm{N}$

\section{Crossover and Mutation:}

Single-point crossover has been used in the proposed system. This crossover point is generated randomly. For mutation, swap method is used followed by fitness calculation. These operators generate a complete new set of population.

\subsection{Weka Tool}

Weka is a landmark system in the history of the data mining and machine learning research communities, because it is the only toolkit that has gained such widespread adoption and 
survived for an extended period of time. The Weka or woodhen (Gallirallus australis) is an endemic bird of New Zealand. It provides many different algorithms for data mining and machine learning. Weka is open source and freely available. It is also platform-independent The GUI Chooser consists of four buttons:

- Explorer: An environment for exploring data with WEKA.

- Experimenter: An environment for performing experiments and conducting statistical tests between learning schemes.

- Knowledge Flow: This environment supports essentially the same functions as the Explorer but with a drag-and-drop interface. One advantage is that it supports incremental learning.

- Simple CLI: Provides a simple command-line interface that allows direct execution of WEKA commands for operating systems that do not provide their own command line interface.

\subsection{Ultrasonic Sensor}

Here we used the Ultrasonic Sensor technique to measure the water level. Ultrasonic sensors are based on the measurement of the properties of acoustic waves with frequencies above the human audible range, often at roughly $40 \mathrm{kHz}$. They typically operate by generating a high-frequency pulse of sound, and then receiving and evaluating the properties of the echo pulse.

An ultrasonic flow meter is a type of flow meter that measures the velocity of a fluid with ultrasound to calculate volume flow. Using ultrasonic transducers, the flow meter can measure the average velocity along the path of an emitted beam of ultrasound, by averaging the difference in measured transit time between the pulses of ultrasound propagating into and against the direction of the flow or by measuring the frequency shift from the Doppler Effect. Ultrasonic flow meters are affected by the acoustic properties of the fluid and can be impacted by temperature, density, viscosity and suspended particulates depending on the exact flow meter.

\section{Conclusion}

In this study, we presented the MEGA initiative for defining reference architecture for water management based on integrating IoT capabilities to achieve a scalable and feasible industrial system algorithm techniques are used to find out the time execution, frequency, accuracy and execution process of water management. High accuracy achieved through weka tool technique compare than $\mathrm{R}$ tool. Time execution achieved $97.50 \%$ sensitivity, $98.90 \%$ frequency, execution process $98.50 \%$ compare than other algorithms. Ultrasonic Sensor is use to find out water $\mathrm{PH}$ Level. In this paper, commonly applying the PH Level measurement test to find out the capacity. Thus the conclusion of this paper produces the result of getting more accuracy through the weka tool in comparison of $\mathrm{R}$ and Orange tool. Finally, Weka tool is best compare than other classification tool. Future work will describe the performed test and will focus on the contribution to solve coordination problems when executing multiple recipes over the same physical resources, considering priority and conditional executions and also process optimization. 


\section{References}

[1] United Nations. Global Sustainable Development Report - Executive Summary: Building the Common Future We Want. New York: United Nations Department of Economic and Social Affairs, Division for Sustainable Development. 2013.

[2] T. Robles, R. Alcarria, D. Mart' in, and A. Morales, "An Internet of Things-based model for smart water management," in Proc. of the 8th International Conference on Advanced Information Networking and Applications Workshops (WAINA'14), Victoria, Canada. IEEE, May 2014, pp. 821-826.

[3] HollandTrade, homepage: http://www.hollandtrade.com/, last viewed November 2014, 2014.

[4] Association of Water Technologies, homepage: http://www.awt.org/, last viewed November 2014, 2014.

[5] EU Water Framework Directive (Directive 2000/60/EC), 2000.

[6] TRAGSA group home page: http://www.tragsa.es/en/, last viewed November 2014.

[7] MEGA project homepage: http://www.gestiondelagua.es/en/, last viewed November 2014.

[8] OPC homepage: https://opcfoundation.org, last viewed November 2014.

[9] J. Gubbi, R. Buyya, S. Marusic, and M. Palaniswami, "Building the common future we want," United Nations Department of Economic and Social Affairs, 2013.

[10] M. Weiser, R. Gold, and J. S. Brown, "The Origins of Ubiquitous Computing Research at PARC in the Late 1980s," IBM Systems Journal, vol. 38, no. 4, pp. 693-696, December 1999.

[11] R. Alcarria, T. Robles, A. Morales, D. L. de Ipina, and U. Aguilera, "Enabling flexible and continuous capability invocation in mobile prosumer environments," Sensors, vol. 12, no. 7, pp. 8930-8954, June 2012.

[12] R. Alcarria, T. Robles, A. Morales, and E. Cedeno, "Resolving coordination challenges in distributed mobile service executions," International Journal of Web and Grid Services, vol. 10, no. 2, pp. 168-191, January 2014.

[13] E. Serrano, P. Moncada, M. Garijo, and C. Iglesias, "Evaluating social choice techniques into intelligent environments by agent based social simulation," Information Sciences, vol. 286, pp. 102-124, December 2014.

[14] L. Atzori, A. Iera, and G. Morabito, "The Internet of Things: A survey," Computer Networks, vol. 54, no. 15, pp. 2787-2805, October 2010.

[15] D. Miorandi, S. Sicari, F. D. Pellegrini, and I. Chlamtac, "Internet of things: Vision, applications and research challenges," Ad Hoc Networks, vol. 10, no. 7, pp. 1497-1516, September 2012.

[16] AENOR EN 61512: Batch control - Part 2: Data structures and guidelines for languages, 2002.

[17] AENOR EN 62264: Enterprise-control system integration - Part 1: Models and terminology, 2013.

[18] M. Melik-Merkumians, T. Baier, M. Steinegger, W. Lepuschitz, I. Hegny, and A. Zoitl, "Towards OPC UA as portable SOA middleware between control software and external added value applications," in Proc. of the 17th IEEE Conference on Emerging Technologies and Factory Automation (ETFA'12), Krak'ow, Poland. IEEE, September 2012, pp. 1-8.

[19] J. Imtiaz and J. Jasperneite, "Scalability of OPC-UA down to the chip level enables Internet of Things," in Proc. of the 11th IEEE International Conference on Industrial Informatics (INDIN'13), Bochum, Germany. IEEE, July 2013, pp. 500-505.

[20] PSI Directive (2003/98/EC). Directive on the re-use of public sector information entered into force on 31 December 2003, European Commission. 